\title{
A Literature Review of Some Deseases Can Cured With Tea Component
}

\author{
Tuti Anggraini \\ \# Technology of Agricultural Product Faculty Agriculture Technology Kampus Limau Manis Andalas University, 25163 Padang West \\ Sumatra Indonesia \\ E-mail: tuty_anggraini@yahoo.co.id
}

\begin{abstract}
Tea (Camellia sinensis) very rich in flavonoids and one of the important plant in the world. The six major flavan-3-ols occurring in tea are catechin (C), gallocatechin (GC), epicatechin (EC), epigallocatechin (EGC), epicatechin-3-gallate (EGG), and epigallocatechin-3-gallate (EGCG). Theaflavins and thearubigins are a group of polyphenol pigments formed during the fermentation of black tea This paper identified the deaseas can cured with the tea properties by literature reviewed. Besides the tea component itself, cancer can prevent with synergyc with capsicum. At a ratio of 25 parts green tea concentrate to 1 part Capsicum preparation, the resultant product exhibited efficacy in the killing of cancer cells in culture 100-times that of green tea on a weight basis. The tea property/polyphenols can use for cancer such as pancreatic cancer, tumor, immortalized keratinocytes and skin cancer cell lines, breast cancer, breaks in bone marrow cells of leukaemic, reduce skin tumor cell, anti-inflammatory, prevent influenza infection in children. Tea pyphenols in black tea nor green tea can reduce human cancer cells, tumor, breast cancer, anti-inflammatory, leukemia and to prevent influenza. EGCG to treat pancreatic cancer, skin cancer, reduce skin tumor cell and keratinocytes. EC for anticancer therapy against acute myeloid leukemia and TF, TR for treating human skin cancer cells.
\end{abstract}

Keywords - Tea; Polyphenol; Cancer.

\section{INTRODUCTION}

Tea (Camellia sinensis) very rich in flavonoids and one of the important plant in the world. Beverages from tea are very popular in the world because its unique taste and flavor. Tea leaves produced by various process depend on the tea product. There were three kinds of tea classyfy from the different processing, green tea, black tea and oolong tea. Green tea is a non enzymatic oxidation, withering to inactivate the enzymes, rolling and drying. Black tea is an enzymatic oxidation by polyphenol substrates to form theaflavin and thearubigin as the final product. Theaflavins are a kind of natural antioxidant. The phenolic hydroxyl group of theaflavins possesses antioxidant activity as a radical scavenger [1]. The processing for black tea were withering, rolling, enzymes oxidation and drying. Oolong tea is a semi enzymatic oxidation. Green tea gives green liquor, black tea gives brown appearance and oolong tea somewhere between black and green tea. Fresh tea leaves contain (on average related to dry substance mass): $36 \%$ polyphenol compounds, $25 \%$ carbohydrates, $15 \%$ proteins, $6.5 \%$ lignin, $5 \%$ ash, $4 \%$ amino acids, $2 \%$ lipids, $1.5 \%$ organic acids, $0.5 \%$ chlorophyll, and carotenoids and volatile substances constituting less than $0.1 \%$ [2].
The six major flavan-3-ols occurring in tea are catechin (C), gallocatechin (GC), epicatechin (EC), epigallocatechin (EGC), epicatechin-3-gallate (EGG), and epigallocatechin-3gallate (EGCG) [3]. The free radicals produced by radiation, chemical reactions, and several redox reactions of various compounds are known to be scavenged by synthetic antioxidants, but due to their adverse side effects leading to carcinogenicity, the search for effective and natural antioxidants has become crucial [4].

This article summarizes the findings of studies using tea component as chemopreventive, natural healing, and antiaging agents for human skin, and discusses. This paper will influence people to consume tea because of the functional properties for human health.

\section{MATERIAL AND MethoD}

\section{Literature Review}

This paper identified some desease that can cured by the component of various tea from some paper.

\section{RESULT AND DISCUSSION}

Tea is rich in flavonoids and other polyphenols that have been shown to have a wide range of biological and 
pharmaceutical benefits, and these polyphenols have recently attracted attention as bioactive agents with anticancer, antidiabetic, antiviral, antimalarial, hepatoprotective, neuroprotective, and cardioprotective effects [5,6] The main catechin group consists of eight polyphenol flavonoid-type compounds: catechin (C), epicatechin (EC), gallocatechin (GC), epigallocatechin (EGC), catechin gallate (CG), epicatechin gallate (ECG), gallocatechin gallate (GCG), and epigallocatechin gallate (EGCG).

\section{A. Anticancer.}

A synergy between a decaffeinated green tea concentrate and a vanilloid-containing Capsicum preparation obtained commercially. At a ratio of 25 parts green tea concentrate to 1 part Capsicum preparation, the resultant product exhibited efficacy in the killing of cancer cells in culture 100-times that of green tea on a weight basis. The activity of the protein target was inhibited by the tea catechins and the Capsicum vanilloids. As with growth, the tea and Capsicum preparations evaluated were synergistic in their inhibition of the target enzymatic activity [7].

EGCG-treated pancreatic cancer decrease cell adhesion ability on micro-pattern dots, accompanied by dephosphorylations of both focal adhesion kinase whereas retained the activations of mitogen-activated protein kinase and mammalian target of rapamycin. At a dose of $100 \mu \mathrm{M}$ EGCG suppresses more than $50 \%$ of cell proliferation without evidence of apoptosis analyzed [8].

The concept that the combination of anticancer drugs with green tea catechin synergistically induces apoptosis of human cancer cells, inhibits tumor formation in mice, and enhances inhibition of tumor growth in xenograft mouse models. As a molecular mechanism by the combination, the induction of growth arrest and DNA damageinducible 153 (GADD153,CHOP) gene expression is discussed in relation to death receptor 5 and TRAILapoptotic pathway. The combination of anticancer drugs with green tea could be a new cancer therapeutic strategy in humans [9].

EGCG is the most effective apoptosis inducing polyphenol present in green tea. They are also in support of our hypothesis that prooxidant action of plant polyphenols may be an important mechanism of their anticancer properties. The rate of oxidative DNA degradation as well as hydroxyl radical and superoxide anion formation was found to be greater in the case of EGCG as compared with EC. It was also shown that copper mediated oxidation of EC and EGCG possibly leads to the formation of polymerized polyphenols. Further, it was indicated that copper oxidized catechins were more efficient prooxidants as compared with their unoxidized forms [10].

The impact of EGCG and AdoHcy hydrolase with 3deazaneplanocin A cotreatment on skin cancer cell function. EGCG and 3-deazaneplanocin A, independently and in combination, reduce the level of polycomb group. The reduction in polycomb group protein level is associated with increased ubiquitination and is reversed by proteasome inhibitors, suggesting proteasome-associated degradation [11].
Compared with nondrinkers, regular drinking of green tea was associated with a slightly decreased risk for breast cancer. Among premenopausal women, reduced risk was observed for years of green tea drinking. Drinking green tea may be weakly associated with a decreased risk of breast cancer [12].

Recently, cytotoxic reactive oxygen species (ROS) were identified in tumor and certain normal cell cultures incubated with high concentrations of the most abundant green tea polyphneol (-)-epigallocatechin-3-gallate (EGCG). If EGCG also provokes the production of ROS in normal epithelial cells, it may preclude the topical use of EGCG at higher doses. The High concentrations of EGCG induced oxidative stress only in tumor cells. In contrast, EGCG reduced ROS in normal cells to background levels. 3-(4,5-dimethylthiazol2-yl)-2,5-diphenyltetrazolium bromide assay and 5bromodeoxyuridine incorporation data were also compared between the two oral carcinoma cell lines treated by EGCG, which suggest that a difference in the levels of endogenous catalase activity may play an important role in reducing oxidative stress provoked by EGCG in tumor cells. Naturally occurring polyphenols to enhance the effectiveness of chemo/radiation therapy to promote cancer cell death while protecting normal cells. Topical and oral administration of green tea polyphenols even at low concentrations such as 15 M, would successfully provide protection against oxidative stress, especially $\mathrm{H} 2 \mathrm{O} 2$, in such tolerant cells. EGCG do not produce $\mathrm{H} 2 \mathrm{O} 2$ in cells derived from the normal epidermis and oral cavity (and possibly digestive tract), but rather protect these cells by decreasing ROS production. Mechanisms responsible for the differential effects of EGCG could rely on distinctive signal pathways activated by EGCG in a tissue-specific manner that requires further investigation [13].

Theaflavins are a group of polyphenol pigments formed during the fermentation of black tea. Antioxidant action of theaflavins is expected to be similar to that of other plant polyphenols, such as catechins, which function as an antioxidant by electron donation to free radical oxidants in aqueous solutions. Theaflavins consist of four major compounds: theaflavin (TF-f), theaflavin 3- gallate (TF-A), theaflavin 3'- gallate (TF-B), and theaflavin 3,3'- digallate (TF-dg) [14]. Theaflavins (TF) and thearubigins (TR) are the most exclusive polyphenols of black tea. Even though few previous reports showed the anticancer effects of TF through apoptosis, the potential effect of TR has not been appraised. Both TF and TR could exert inhibition of A431 (human epidermoid carcinoma) and A375 (human malignant melanoma) cell proliferation without adversely affecting normal human epidermal keratinocyte cells. Furthermore, TF and TR elicited intracellular TF as well as TR might exert chemopreventive effect through cell cycle arrest and induction of apoptogenic signals via mitochondrial death cascade in human skin cancer cells. The induction of apoptosis and its mechanism is induced by TR, an important polyphenol exclusively present in black tea [15].

The liver enzyme, xanthine oxidase (XO) produces uric acid and reactive oxygen species (ROS) during the catabolism of purines.. Polyphenols are antioxidants, and it has been suggested that they can reduce oxidative stress by their antioxidant properties. Tea catechins may act at.an 
earlier stage than has previously been suspected, by inhibiting ROS production, rather than only neutralizing the already formed ROS. This suggests a new mechanism whereby tea drinking may prevent oxidative stress related diseases, e.g. atherosclerosis and cancer [16].

\section{B. Influenza}

Green tea is known to contain antiviral components that prevent influenza infection. Anonymous questionnaire surveys were undertaken twice during the influenza season from November 2008 to February 2009 (endemic seasonal type A influenza infection); each survey was conducted for 2663 pupils across all elementary schools in Kikugawa City (a tea plantation area), Japan. Each questionnaire was completed and submitted by 2050 pupils (response rate, $77.0 \%$; age range, 6-13 y). The adjusted OR associated with the consumption of green tea for $\$ 6 \mathrm{~d} / \mathrm{wk}$ compared with ,3 $\mathrm{d} /$ wk was $0.60[(95 \% \mathrm{CI}=0.39-0.92) ; \mathrm{P}=0.02]$ in cases of influenza confirmed by the antigen test. Meanwhile, the adjusted OR inversely associated with the consumption of 1 cup $/ \mathrm{d}$ to,3 cups $/ \mathrm{d}$ (1 cup $=200 \mathrm{~mL})$ and $3-5 \mathrm{cups} / \mathrm{d}$ compared with, $1 \mathrm{cup} / \mathrm{d}$ were 0.62 [ $(95 \% \mathrm{CI}=0.41-0.95) ; \mathrm{P}$ $=0.03]$ and 0.54 [(95\% CI $=0.30-0.94) ; \mathrm{P}=0.03]$, respectively. However, there was no significant association with the consumption of $.5 \mathrm{cups} / \mathrm{d}$. Our findings thus suggest that the consumption of $1-5$ cups/d of green tea may prevent influenza infection in children [17].

\section{Leukemia}

Anti-proliferation and apoptosis inducing effects of were studied against human promyeolcytic leukemia HL-60 cells. Green tea polyphenols significantly reduced the HL-60 proliferation, however, they did not show significant inhibition in proliferation of normal V79-4 cells. Green tea polyphenol treatment induced apoptosis in HL-60 cells, evidenced by nuclei fragmentation and apoptotic body appearance. Green tea polyphenols revealed antiproliferative and apoptosisinducing activity against HL-60 cells through the down regulation of $\mathrm{Bcl}-2$ and activation of caspase-3 [18].

The polycomb group (PcG) proteins are epigenetic regulators of gene expression that enhance cell survival. The increased expression of key PcG proteins in immortalized keratinocytes and skin cancer cell lines. EGCG treatment of SCC-13 cells reduces Bmi-1 and Ezh2 level and this is associated with reduced cell survival. The reduction in survival is associated with a global reduction in histone $\mathrm{H} 3$ lysine 27 trimethylation, a hallmark of PRC 2 complex action. Green tea polyphenols reduce skin tumor cell survival by influencing PcG-mediated epigenetic regulatory mechanisms [19].

Epicatechin (EC) induces oxidative DNA damage in HL60 cells. The association between genotoxic and apoptotic/necrotic effects of EC was studied in rats with acute myeloid leukaemia. EC significantly increased DNA strand breaks in bone marrow cells of leukaemic animals but it did not exert such an effect on healthy rats. EC action led to necrosis of leukaemia cells but it did not induce apoptosis of these cells in comparison to the controls. Conclusion: EC has genotoxic and necrotic effects which may have utility in anticancer therapy against acute myeloid leukaemia [20].

\section{Keratinocytes}

The most abundant green tea polyphenol, epigallocatechin-3-gallate (EGCG), was found to induce differential effects between tumor cells and normal cells. Tea polyphenols may be used for treatment of wounds or certain skin conditions characterized by altered cellular activities or metabolism. EGCG or a mixture of the major green tea polyphenols stimulated aged keratinocytes to generate biological energy and to synthesize DNA, possibly for renewed cell division., green tea constituents may be useful topically for promoting skin regeneration, wound healing, or treatment of certain epithelial conditions such as aphthous ulcers, psoriasis, rosacea, and actinic keratosis. In addition, the differentiation-inducing potential of green tea components might be beneficial to patients who have conditions characterized by abnormally accelerated skin cell growth and lack of differentiation [21].
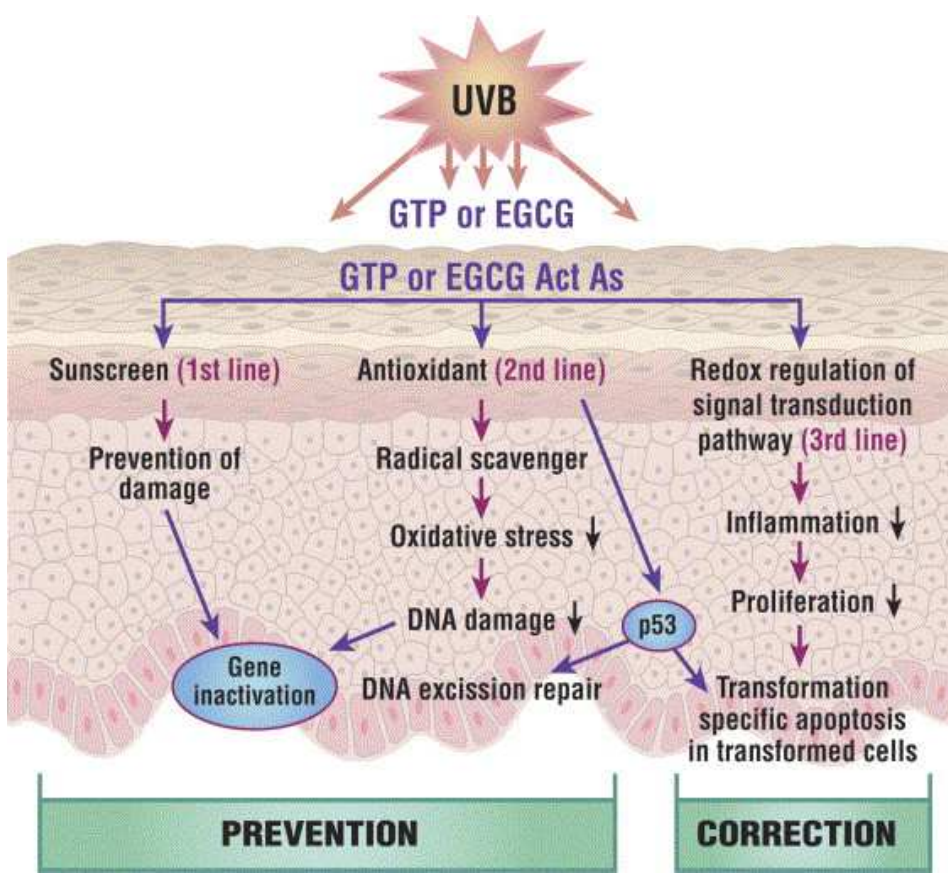

Figures from [21]

Fig 1. Schematic illustration of properties of green tea in photoprotection against UV irradiation Only epidermis is shown. EGCG, (-)Epigallocatechin-3-gallate; GTP, green tea polyphenol

\section{E. Antiimflammatory}

Green tea polyphenols are potent antioxidants. However, their mechanisms of actions remain unclear. In inflammation, tumor necrosis factor- $\alpha(\mathrm{TNF} \alpha)$ plays a pivotal role., ()epigallocatechin gallate (EGCG), the major green tea polyphenol, decreased lipopolysaccharide (LPS)-induced $\mathrm{TNF} \alpha$ production in a dose-dependent fashion (50\% 
inhibition at $100 \mathrm{mmol} / \mathrm{L})$. EGCG also inhibited LPSinduced TNF $\alpha$. EGCG inhibited LPS-induced TNF $\alpha$ production in elicited mouse peritoneal macrophages. In male BALB/c mice, green tea polyphenols (given by oral gavage $2 \mathrm{~h}$ prior to an . injection of $40 \mathrm{mg} \mathrm{LPS} / \mathrm{kg}$ body wt) decreased LPS-induced TNF $\alpha$ production in serum in a dose-responsive fashion. At a dose of $0.5 \mathrm{~g}$ green tea polyphenols/kg body wt, serum TNF $\alpha$ was reduced by $80 \%$ of control. Moreover, $0.5 \mathrm{~g}$ green tea polyphenols $/ \mathrm{kg}$ body wt completely inhibited LPS-induced lethality in male $\mathrm{BALB} / \mathrm{c}$ mice. Green tea polyphenols may be effective therapy for a variety of inflammatory processes A $70 \mathrm{~kg}$ human would have to consume nearly 300 cups (30 L) of green tea to achieve a dose equivalent to that given to our mice in the lethality experiments. However, concentrated forms of green tea extracts are now being sold as natural supplements through many sources [22]. The induction of SOCS1(suppressor of cytokine signaling 1) gene expression by EGCG may play a central role in the anti-inflammatory function of EGCG [23].

The chemotherapeutic agent irinotecan (IT) is highly effective against several types of cancer, although its use is limited due to severe intestinal toxicity. Tea polyphenols could ameliorate the detrimental effects induced by IT. In Expt. 1, mice were challenged intraperitoneally with IT or saline on 2 consecutive days. For time kinetic measurements, the IT-treated mice were killed at 3, 24, 48, 72, and $96 \mathrm{~h}$ after the 2nd dose of IT. Three hours after IT administration, the ileum glutathione concentration dropped significantly. Lipid peroxidation and inflammation, as measured by macrophage inflammatory protein-2 content, myeloperoxidase activity In Expt. 2, green tea polyphenols $(1 \mathrm{~g} / \mathrm{L})$ were supplied via drinking water for $7 \mathrm{~d}$ before and 3 d after treatment with IT. Green tea polyphenols significantly affected the glutathione:glutathione disulfide ratio but not lipid peroxidation, macrophage inflammatory protein-2 levels, myeloperoxidase activity. IT administration is associated with oxidative stress and inflammation, both occurring simultaneously to IT-induced mucosal damage. The antioxidative defense is affected soon after IT administration. Green tea polyphenols supplied orally protected against oxidation and could be one approach to reducing the risk of IT-induced side effects in the clinical setting. oxidative as well as inflammatory processes are involved in the IT-induced destruction of small intestinal epithelium in healthy mice [24].

\section{CONCLUSION}

The tea property/polyphenols can use for cancer such as pancreatic cancer, tumor, immortalized keratinocytes and skin cancer cell lines, breast cancer, breaks in bone marrow cells of leukaemic, reduce skin tumor cell, anti-inflammatory, prevent influenza infection in children. Tea pyphenols in black tea nor green tea can reduce human cancer cells, tumor, breast cancer, anti-inflammatory, leukemia and to prevent influenza. EGCG to treat pancreatic cancer, skin cancer, reduce skin tumor cell and keratinocytes. EC for anticancer therapy against acute myeloid leukemia and TF, TR for treating human skin cancer cells.

\section{REFERENCE}

[1] Yoshino K, Yamazaki K, Sano M. 2010. Preventive effects of black tea theaflavins against mouse type IV allergy. Journal of the Science of Food and Agriculture Volume 90, Issue 12, pages 1983-1987

[2] Harold NG and Graham. 1992. Green tea composition, consumption and polyphenol chemistry. Preve Med . 21:334-350.

[3] Robertson, A. 1992. The chemistry and biochemistry of black tea production-the non volatiles, in Tea : Cultivation to consumption, Ed by Willson A and Clifford M N, Chapman and Hall. London, 555901.

[4] Choi, Y., Jeong, H-S., and Lee, J. 2006. Antioxidant activity of metahnolic extracts from some grains consumed in Korea. Food Chemistry 103: 130-138.

[5] Adhami, V.M., Malik, A., Zaman, N., Sarfarz, S., Siddiqui, I.A., Syed, D.N., Afaq, F., Pasha, F.S., Saleem, M., and Mukhtar, H. 2007. Combined inhibitory effects of green tea polyphenols and selective cyclooxygenase- 2 inhibitors on the growth of human prostate cancer cells both in vitro and in vivo. Clinical Cancer Research 13, 16111619 .

[6] Anderson, R.A., and Polansky, M.M. 2002. Tea enhances insulin activity. Journal of Agriculture and Food Chemistry 50 7182-7186.

[7] Morre J and Morre D. 2010. Synergistic Capsicum-tea mixtures with anticancer activity. Journal of Pharmacy and Pharmacology.Volume 55, Issue 7, pages 987-994.

[8] Vu HA, Beppu Y, Thanh H, Sasaki K, Yamamoto H, Xinh PT, Tanii T, Hara Y, Watanabe T, Sato Y and Ohdomari. 2010. Green Tea Epigallocatechin Gallate Exhibits Anticancer Effect in Human Pancreatic Carcinoma Cells via the Inhibition of Both Focal Adhesion Kinase and Insulin-Like Growth Factor-I Receptor. Journal of Biomedicine and Biotechnology Volume 2010.

[9] Fujiki H and Suganuma M. 2012. Green tea: An effective synergist with anticancer drugs for tertiary cancer prevention. Cancer Letters. Volume 324, Issue 2, 28 November 2012, Pages 119-125.

[10] Azam S, Hadi N, Khan NU and Hadi SM. 2004. Prooxidant property of green tea polyphenols epicatechin and epigallocatechin-3-gallate: implications for anticancer properties Toxicology in Vitro. Volume 18, Issue 5, October 2004, Pages 555-561.

[11] Choudhury SR, Balasubramanian S, Chew YC, Han B, Marquez VE and Eckert RL. 2011. (-)-Epigallocatechin-3-gallate and DZNep reduce polycomb protein level via a proteasome-dependent mechanism in skin cancer cells. Carcinogenesis vol.32 no.10 pp.1525-1532.

[12] Shrubsole MJ, Lu W, Chen Z, Shu XO, Zheng Y, Dai Q, Cai Q, Gu K, Ruan ZX, Gao YT and Zheng W. 2009. Drinking Green Tea Modestly Reduces Breast Cancer Risk. J. Nutr. February 2009vol. 139 no. 2 310-316.

[13] Yamamoto T, Hsu S, Lewis J, Wataha J, Dickinson D, Singh B, Bollag WB, Lockwood P, UetaE, Osaki T and Schuster G. 2003. Green Tea Polyphenol Causes Differential Oxidative Environments in Tumor versus Normal Epithelial Cells. THE JOURNAL OF PHARMACOLOGY AND EXPERIMENTAL THERAPEUTICS. JPET October 2003 vol. 307 no. 1 230-236.

[14] Chunxia Wang and Yongquan Li. 2005. Research progress on property and application of theaflavins African Journal of Biotechnology Vol. 5 (3), pp. 213-218.

[15] Halder B, Bhattacharya U, Mukhopadhay and Giri AK. 2008. Molecular mechanism of black tea polyphenols induced apoptosis in human skin cancer cells: involvement of Bax translocation and mitochondria mediated death cascade. Carcinogenesis vol.29 no.1 pp.129-138.

[16] Aucam J, Gaspa A, Hara Y and Apostolides Z. 1997. Inhibition of xanthine oxidase by catechins from tea (Camellia sinensis). Anticancer Research [1997, 17(6D):4381-4385.

[17] Park M, Yamada H, Matshushita K, Kaji S, Goto T, Okada Y, Kosuge $\mathrm{K}$ and Kitagawa T. 2011. Green Tea Consumption Is Inversely Associated with the Incidence of Influenza Infection among Schoolchildren in a Tea Plantation Area of Japan. J. Nutr. October 1, 2011 vol. 141 no. $101862-1870$.

[18] Han DH, Jeong JH and Kim JH. 2009. Anti-proliferative and Apoptosis Induction Activity of Green Tea Polyphenols on Human Promyelocytic Leukemia HL-60 Cells. ANTICANCER RESEARCH 29: 1417-1422.

[19] Balasubramanian S, Adhikary G and Eckert RL. 2010. The Bmi-1 polycomb protein antagonizes the (2)-epigallocatechin-3-gallate- 
dependent suppression of skin cancer cell survival. Carcinogenesis vol.31 no.3 pp.496-503.

[20] Papiez MA, Baran J, Strakova KB and Krosniak M. 2011 Epicatechin Administration Leads to Necrotic Cell Death of Rat Leukaemia Promyelocytes In Vivo. In Vivo January-February 2011 vol. 25no. $129-34$.

[21] Hsu S, Bollag WB, Lewis J, Huang Q, Singh B, Sharawy M, Yamamoyo T and Shuster G. 2003. Green Tea Polyphenols Induce Differentiation and Proliferation in Epidermal Keratinocytes. THE JOURNAL OF PHARMACOLOGY AND EXPERIMENTAL THERAPEUTICS. Vol. 306, No. 1.

[22] Yang F, Villiers WJS, McClain CJ, Varilek GW. 1998. Green Tea Polyphenols Block Endotoxin-Induced Tumor Necrosis FactorProduction and Lethality in a Murine Model. J. Nutr. December 1, 1998 vol. 128 no. $122334-2340$

[23] Ripley BJM, Fujimoto M, Serada S, Ohkawara T, Nishikawa T, Terabe F, Matsukawa Y, Stephanou A, Knight R, Isenberg DA, Latchman DS, Kishimoto T and Naka T. 2010. Green tea polyphenol epigallocatechin gallate inhibits cell signaling by inducing SOCS1 gene expression. International Immunology, Vol. 22, No. 5, pp. 359366

[24] Wessner B, Strasser EM, Koitz Nina, Schuckenschlager C, Manhart NU and Roth E. 2007. Green Tea Polyphenol Administration Partly Ameliorates Chemotherapy-Induced Side Effects in the Small Intestine of Mice. J. Nutr. March 2007 vol. 137 no. 3 634-640. 